\title{
Analgesia Pós-Operatória para Crianças com Menos de 1 Ano. Análise Retrospectiva *
}

\section{Postoperative Analgesia in Children Less than 1 Year of Age. A Retrospective Analysis}

\author{
Paulo do Nascimento Junior, TSA ${ }^{1}$, Norma Sueli Pinheiro Módolo, TSA ${ }^{1}$, Geraldo Rolim Rodrigues Junior, TSA ${ }^{1}$
}

\section{RESUMO}

Nascimento Jr P, Módolo NSP, Rodrigues Jr GR - Analgesia Pós-Operatória para Crianças com Menos de 1 Ano. Análise Retrospectiva

Justificativa e Objetivos - A dor pós-operatória continua sendo uma das principais complicações pós-operatórias e motivo de desconforto, principalmente em crianças. O objetivo deste estudo foi avaliar o uso de analgésicos desde o término da cirurgia até a alta da sala de recuperação pós-anestésica (SRPA), como medida terapêutica ou profilática, para crianças com menos de 1 ano de idade.

Método - Utilizando o banco de dados do Departamento de Anestesiologia, foi realizada análise retrospectiva, envolvendo o período de janeiro de 2000 a abril de 2001, das anestesias de crianças menores que 1 ano de idade submetidas a procedimentos cirúrgicos diversos, avaliando aspectos relacionados à analgesia pós-operatória.

Resultados - No período do estudo, foram anestesiadas 402 crianças menores que 1 ano, sendo que 194 (48,2\%) não receberam analgésicos e 208 (51,8\%) receberam. Com relação ao uso ou não de analgésicos, foi observado o que se segue: Sem analgésicos: (1) Idade: até 1 mês, 68/99; entre 1 e 6 meses, 53/126; entre 6 meses e 1 ano, 73/177. (2) Peso: 6,7 \pm $3,1 \mathrm{~kg}$ (3). Sexo: masculino, 106/240; feminino, 88/162. (4) Estado físico ASA: ASA I, 69/187; ASA II, 56/113; ASA III, 46/79; ASA IV, 23/23. (5) Anestesia peridural sacral: 3/4. (6) Tempo de anestesia: $106 \pm 32$ minutos. (7) Encaminhamento para unidade de terapia intensiva (UTI): 93/119. Uso de analgésicos: (1) Idade: até 1 mês, 31/99; entre 1 e 6 meses, 73/126; entre 6 meses e 1 ano, 104/177. (2) Peso: $9 \pm 2,3 \mathrm{~kg}$. (3) Sexo: masculino, 134/240; feminino, 74/162. (4) Estado físico ASA: ASA I, 118/187; ASA II, 57/113; ASA III, 33/79; ASA IV, 0/23. (5) Anestesia peridural sacral: 1/4. (6) Tempo de anestesia: $130 \pm 38$ minutos. (7) Encaminhamento para UTI: 26/119. Os fármacos empregados para promover analgesia foram: dipirona $(60,6 \%)$, dipirona + tramadol $(25,5 \%)$, dipirona + nalbufina $(5,3 \%)$, tramadol $(3,8 \%)$, nalbufina $(3,8 \%)$, meperidina $(0,5 \%)$ e fentanil $(0,5 \%)$.

Conclusões - Utilizar analgésicos em crianças desde o término da cirurgia até a alta da SRPA não foi habitual, principalmente nas crianças menores e mais graves e em

\footnotetext{
${ }^{*}$ Recebido do (Received from) Departamento de Anestesiologia da Faculdade de Medicina de Botucatu (FMB - UNESP), Botucatu, SP

1. Professor Assistente Doutor do Departamento de Anestesiologia da FMB - UNESP

Apresentado (Submitted) em 03 de dezembro de 2001

Aceito (Accepted) para publicação em 19 de abril de 2002

Correspondência para (Mail to)

Dr. Paulo do Nascimento Júnior

Dept ${ }^{\circ}$ de Anestesiologia da FMB - UNESP

Distrito de Rubião Junior

18618-970 Botucatu, SP

E-mail:pnasc@fmb.unesp.br

(C) Sociedade Brasileira de Anestesiologia, 2002
}

procedimentos cirúrgicos mais rápidos. O uso de dipirona, isoladamente, ou a associação dipirona/tramadol, foram as drogas analgésicas mais freqüentemente empregadas.

UNITERMOS: ANESTESIA, Pediátrica; DOR, Aguda: pós-operatória

\section{SUMMARY}

Nascimento Jr P, Módolo NSP, Rodrigues Jr GR - Postoperative Analgesia in Children Less than 1 Year of Age. A Retrospective Analysis

Background and Objectives - Postoperative pain is still a major complication causing discomfort, especially for children. This study aimed at evaluating the use of analgesics from surgery completion to postoperative intensive care unit (PACU) discharge as a prophylactic or therapeutic approach for children less than 1 year of age.

Methods - Based on the Anesthesiology Department files, a retrospective analysis was performed with children less than 1 year of age submitted to different surgical procedures from January/2000 to April/2001, to evaluate postoperative analgesia-related aspects.

Results - During the study period, 402 children aged less than 1 year were anesthetized. From those, 194 (48.2\%) were not medicated with analgesics and 208 (51.8\%) were. As to using or not analgesics, the following was observed: Without analgesics: (1) Age: up to 1 month, 68/99; between 1 and 6 months, 53/126; from 6 months to 1 year, 73/177. (2) Weight: $6.7 \pm 3.1 \mathrm{~kg}$. (3) Gender: male, 106/240; female, 88/162. (4) ASA Physical Status: ASA I, 69/187; ASA II, 56/113; ASA III, 46/79; ASA IV, 23/23. (5) Caudal anesthesia: 3/4. (6) Anesthesia duration: $106 \pm 32$ minutes. (7) Referral to intensive care unit (ICU): 93/119. With analgesics: (1) Age: up to 1 month, 31/99; between 1 and 6 months, 73/126; from 6 months to 1 year, 104/177. (2) Weight: 9 $\pm 2.3 \mathrm{~kg}$. (3) Gender: male, 134/240; female, 74/162. (4) ASA Physical Status: ASA I, 118/187; ASA II, 57/113; ASA III, 33/79; ASA IV, 0/23. (5) Caudal anesthesia: 1/4. (6) Anesthesia duration: $130 \pm 38$ minutes. (7) Referral to ICU: 26/119. Analgesic drugs used were: dipyrone (60.6\%), dipyrone + tramadol (25.5\%), dipyrone + nalbuphine (5.3\%), tramadol (3.8\%), nalbuphine (3.8\%), meperidine (0.5\%) and fentanyl $(0.5 \%)$.

Conclusions - Analgesics prescription for children, from surgery completion to PACU discharge, was not usual, especially in younger and more severely ill children and in shorter surgical procedures. Dipyrone, alone, and the association dipyrone/tramadol were the most frequent drugs used.

KEY WORDS: ANESTHESIA, Pediatric; PAIN, Acute: postoperative

\section{INTRODUÇÃO}

$\Lambda$ dor talvez seja um dos mais temíveis sintomas de doença no ser humano, tendo recebido indevida atenção, até há pouco, na prática médica. Trata-se de um dos problemas mais comuns experimentados por indivíduos em todas as idades ${ }^{1}$. 
O uso de fármacos analgésicos para tratamento da dor pós-operatória em crianças, principalmente nas menores de um mês, é assunto ainda bastante controverso. A resistência em prescrever analgesia pós-operatória adequada a pacientes pediátricos, bem como as razões que justificam este fato, são discutidas na literatura ${ }^{2,3}$. Alguns médicos ainda podem acreditar que os neonatos não sentem dor ou apresentam a percepção da dor alterada. Outros aceitam que as crianças muito pequenas percebem e sentem dor, no entanto preocupam-se com os riscos de prescrever drogas analgésicas potentes ${ }^{4}$.

Uma das dificuldades no adequado manuseio da dor em pediatria refere-se à dificuldade de se obter uma medida acurada, objetiva e quantitativa da dor, principalmente em recém-nascidos, lactentes e pré-escolares, pois a maioria dos métodos atualmente disponíveis não se aplicam a todas as faixas etárias pediátricas ${ }^{5}$

Sabendo-se que a dor pós-operatória continua sendo uma das principais complicações pós-operatórias e motivo de desconforto, principalmente em crianças, e com o intuito de conhecer o perfil das crianças que não recebem analgésicos no período pós-operatório imediato, seja por medida terapêutica ou profilática, o objetivo deste estudo foi avaliar o uso de analgésicos desde o término da cirurgia até a alta da sala de recuperação pós-anestésica (SRPA) em crianças menores que 1 ano, no Hospital das Clínicas da Faculdade de Medicina de Botucatu - UNESP.

\section{MÉTODO}

Utilizando o banco de dados do Departamento de Anestesiologia (Microsoft Access), foi realizada análise retrospectiva, envolvendo o período de janeiro de 2000 a abril de 2001 , de todas as anestesias realizadas em crianças menores que 1 ano de idade, submetidas a procedimentos cirúrgicos diversos e que foram recuperadas na SRPA. Os aspectos relacionados à analgesia pós-operatória foram avaliados considerando-se a porcentagem de pacientes que recebeu fármaco para tratamento de dor no período desde o término da cirurgia até a alta da SRPA, a prevalência de analgesia segundo a idade, o sexo, o peso, a técnica anestésica empregada (realização de anestesia geral ou anestesia peridural sacral) e a situação clínica das crianças, através da determinação do estado físico (ASA) e do destino dos pacientes após a saída da SRPA - unidade de terapia intensiva (UTI) ou enfermaria.

Para a análise da utilização de fármacos analgésicos segundo a idade, dividimos os pacientes em três subgrupos de faixas etárias: crianças com menos de um mês, idades entre $1 \mathrm{e}$ 6 meses e 6 meses e 1 ano.

A clínica de origem dos pacientes também foi pesquisada. A análise estatística foi realizada utilizando-se o teste do Qui-quadrado e o teste Exato de Fisher para verificação de freqüências e o teste $t$ de Student para comparação das variáveis paramétricas.

\section{RESULTADOS}

No período do estudo foram anestesiadas 402 crianças menores que 1 ano de idade, sendo que 194 (48,2\%) não receberam analgésicos e 208 (51,8\%) receberam (Tabela I). A distribuição dos pacientes segundo a idade, que receberam ou não analgésicos, é apresentada na tabela II e na figura 1. A distribuição dos pacientes segundo o sexo e o estado físico (ASA), que receberam ou não analgésicos, é ilustrada nas figuras $2 \mathrm{e}$ 3 , respectivamente, e na tabela II. A avaliação dos pacientes que receberam ou não analgésicos segundo o peso, a técnica anestésica empregada (realização de anestesia geral ou anestesia peridural sacral), duração da anestesia e encaminhamento ou não para a UTI está destacada na tabela II.

Tabela I - Número Total e Respectiva Porcentagem de Pacientes que Receberam ou Não Analgésicos desde o Término da Cirurgia até a Alta da SRPA

\begin{tabular}{lcc}
\hline & Número & $\%$ \\
\hline Sem analgesia & 194 & 48,2 \\
Uso de analgésicos & 208 & 51,8 \\
\hline
\end{tabular}

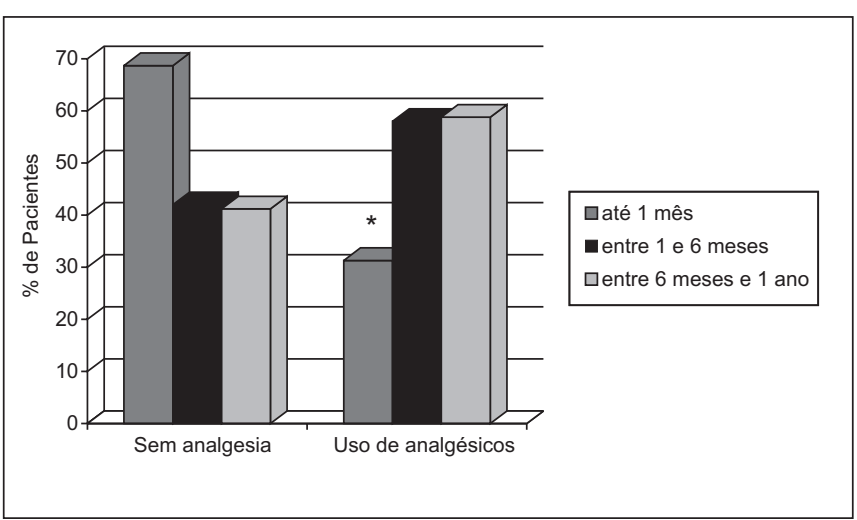

Figura 1 - Distribuição dos Pacientes, em Porcentagem, Segundo a Idade, que Receberam ou Não Analgésicos desde o Término da Cirurgia até a Alta da SRPA $* p<0,01$

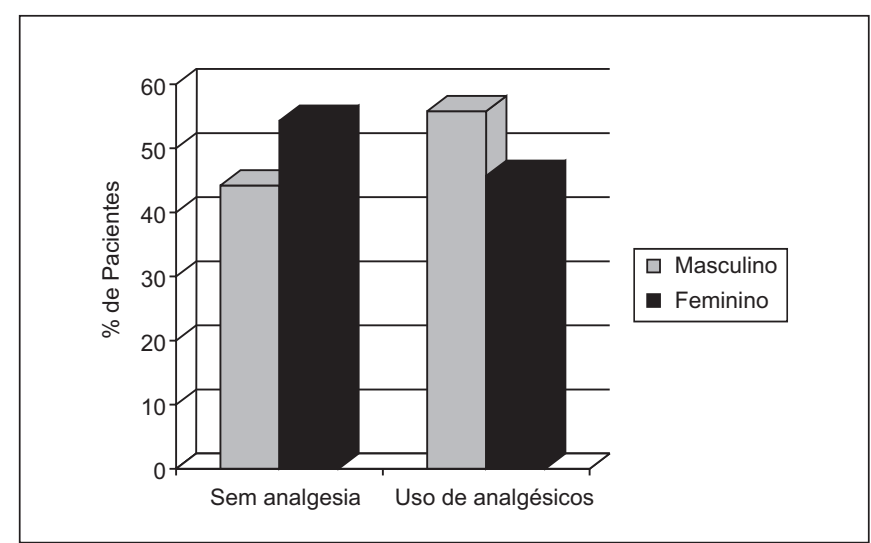

Figura 2 - Distribuição dos Pacientes, em Porcentagem, Segundo o Sexo, que Receberam ou Não Analgésicos desde o Término da Cirurgia até a Alta da SRPA

Revista Brasileira de Anestesiologia Vol. 52, № 6 , Novembro - Dezembro, 2002 
Tabela II - Avaliação dos Pacientes que Receberam ou Não Analgésicos desde o Término da Cirurgia até a Alta da SRPA segundo a Idade, o Sexo, o Estado Físico (ASA), o Peso, a Realização de Anestesia Peridural Sacral, a Duração da Anestesia e o Encaminhamento ou Não para a UTI

\begin{tabular}{|c|c|c|c|}
\hline & Sem analgesia & Uso de analgésicos & Estatística \\
\hline Idade & & & $p<0,01$ \\
\hline Até 1 mês $(n=99)$ & $68 / 99(68,7 \%)$ & $31 / 99(31,3 \%)$ & \\
\hline Entre 1 e 6 meses $(n=126)$ & $53 / 126(42 \%)$ & $73 / 126(58 \%)$ & \\
\hline Entre 6 meses e 1 ano $(n=177)$ & $73 / 177(41,2 \%)$ & $104 / 177(58,8 \%)$ & \\
\hline Sexo & & & $p>0,05$ \\
\hline Masculino $(n=240)$ & $106 / 240(44,2 \%)$ & $134 / 240(55,8 \%)$ & \\
\hline Feminino $(n=162)$ & $88 / 162(54,3 \%)$ & $74 / 162(45,7 \%)$ & \\
\hline Estado físico & & & $p<0,01$ \\
\hline ASA I $(n=187)$ & $69 / 187(36,9 \%)$ & $118 / 187(63,1 \%)$ & \\
\hline ASA $\|(n=113)$ & $56 / 113(49,5 \%)$ & $57 / 113(50,5 \%)$ & \\
\hline ASA III $(n=79)$ & $46 / 79(58,2 \%)$ & $33 / 79(41,8 \%)$ & \\
\hline ASA IV $(n=23)$ & $23 / 23(100 \%)$ & $0 / 23(0 \%)$ & \\
\hline Peso $(\mathrm{kg})^{*}$ & $6,7 \pm 3,1$ & $9,0 \pm 2,3$ & $p>0,05$ \\
\hline Tipo de anestesia & & & Não aplicada \\
\hline Peridural sacral $(n=4)$ & $3 / 4(75 \%)$ & $1 / 4(25 \%)$ & \\
\hline Geral $(n=398)$ & $191 / 398(48 \%)$ & $207 / 398(52 \%)$ & \\
\hline Duração da anestesia $(\mathrm{min})^{*}$ & $106 \pm 21$ & $130 \pm 38$ & $p<0,05$ \\
\hline Paciente encaminhado para UTI $(n=119)$ & $93 / 119(78,1 \%)$ & $26 / 119(21,9 \%)$ & $p<0,01$ \\
\hline
\end{tabular}

* Valores expressos pela Média \pm DP

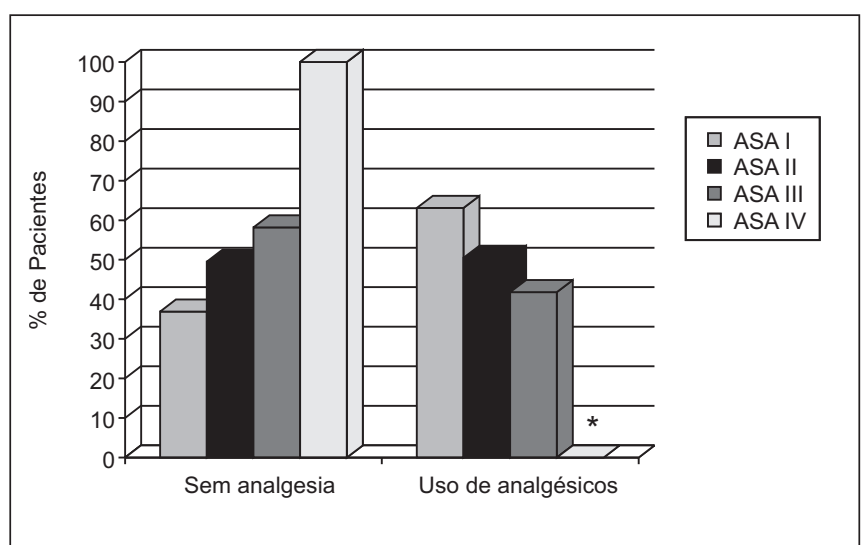

Figura 3 - Distribuição dos Pacientes, em Porcentagem, Segundo o Estado Físico (ASA), que Receberam ou Não Analgésicos desde o Término da Cirurgia até a Alta da SRPA

${ }^{*} p<0,01$

A tabela III apresenta, de acordo com a clínica de origem dos pacientes, a porcentagem dos que receberam ou não analgésicos até a alta da SRPA.

A relação dos fármacos empregados nos 208 pacientes que receberam drogas analgésicas é apresentada na tabela IV.

\section{DISCUSSÃO}

O alívio da dor é o objetivo maior da Anestesiologia. O procedimento anestésico deve contemplar não somente o período per-operatório, mas também o período pós-operatório. A avaliação do controle da dor pós-operatória na criança reve-

Revista Brasileira de Anestesiologia

Vol. 52, № 6 , Novembro - Dezembro, 2002
Tabela III - Pacientes que Receberam ou Não Analgésicos desde o Término da Cirurgia até a Alta da SRPA segundo sua Clínica de Origem

\begin{tabular}{lcc}
\hline & Sem analgesia & Uso de analgésicos \\
\hline Cirurgia pediátrica $(n=167)$ & $76(45,5 \%)$ & $91(54,5 \%)$ \\
Neurocirurgia $(n=66)$ & $27(41 \%)$ & $39(59 \%)$ \\
Oftalmologia $(n=44)$ & $21(47,7 \%)$ & $23(52,3 \%)$ \\
Urologia $(n=27)$ & $7(26 \%)$ & $20(74 \%)$ \\
Otorrinolaringologia $(n=27)$ & $19(70,3 \%)$ & $8(29,7 \%)$ \\
Ortopedia $(n=26)$ & $14(53,8 \%)$ & $12(46,2 \%)$ \\
Cirurgia cardíaca $(n=21)$ & $16(76,1 \%)$ & $5(23,9 \%)$ \\
Cirurgia plástica $(n=17)$ & $8(47 \%)$ & $9(53 \%)$ \\
Cirurgia torácica $(n=7)$ & $6(85,7 \%)$ & $1(14,3 \%)$ \\
\hline
\end{tabular}

Tabela IV - Fármacos Empregados para Promover Analgesia Pós-Operatória desde o Término da Cirurgia até a Alta da SRPA, em Número de Pacientes e Respectiva Porcentagem

\begin{tabular}{lcc}
\hline & Número & $\%$ \\
\hline Dipirona & 126 & 60,6 \\
Associação dipirona/tramadol & 53 & 25,5 \\
Associação dipirona/nalbufina & 11 & 5,3 \\
Tramadol & 8 & 3,8 \\
Nalbufina & 8 & 3,8 \\
Meperidina & 1 & 0,5 \\
Fentanil & 1 & 0,5 \\
\hline
\end{tabular}


lou o atual estado do controle da dor em pediatria ${ }^{6}$. A crença de que as crianças, principalmente os neonatos, não são capazes de sentir dor, tem motivado cirurgiões e anestesiologistas a não prescreverem drogas, especialmente opióides, no período pós-operatório ${ }^{4}$.

O emprego de analgésicos para o tratamento da dor pós-operatória que já se estabeleceu, ou como medida profilática, impedindo que os pacientes sintam qualquer dor, tem grande importância em função da determinação do bem-estar e do conforto no ambiente hospitalar.

Observamos em nossa pesquisa que, dentre as 402 crianças menores que 1 ano de idade estudadas, 194 (48,2\%) não receberam analgésicos no período desde o término da cirurgia até a alta da SRPA. Outros autores também verificaram baixa porcentagem de uso de analgésicos no período pós-operatório, principalmente opióides em neonatos ${ }^{7}$. Também, quando há comparação entre crianças e adultos, existe muita diferença em favor dos adultos com relação à quantidade de analgésicos prescritos no período pós-operatório ${ }^{6}$.

Purcell-Jones e col., em 1988, verificaram que, em procedimentos cirúrgicos de grande porte realizados em neonatos, $48 \%$ dos anestesiologistas não prescreviam opióides para tratamento da dor pós-operatória. No caso de crianças até um ano de idade, $37 \%$ dos anestesiologistas também não empregavam esses fármacos para alívio da dor pós-operatória. Já, em procedimentos cirúrgicos de menor porte, essas porcentagens foram $81 \%$ e $70 \%$, respectivamente, para os mesmos grupos etários ${ }^{4}$. Em análises semelhantes à realizada por Purcell-Jones e col., outros pesquisadores observaram modificação do padrão de realização de analgesia pós-operatória em pacientes pediátricos, com favorecimento das crianças menores ${ }^{8,9}$.

Na nossa casuística, observamos que, para as crianças que receberam fármacos para tratamento da dor pós-operatória, houve menor porcentagem de uso de analgésicos nas menores de 1 mês de idade (Tabela II e Figura 1). Apesar de não haver diferença estatisticamente significante, a média de peso dos pacientes que não receberam analgésicos foi menor que a dos que receberam (Tabela II). Assim, verificamos desfavorecimento dos pacientes menores em relação à prescrição de analgésicos no período pós-operatório imediato.

Quanto às condições clínicas dos pacientes, analisadas através da classificação do estado físico (ASA), obtivemos menor freqüência de uso de analgésicos para as crianças mais graves (ASA III e IV). Nenhum paciente estado físico ASA IV recebeu medicação analgésica ao final da cirurgia ou na SRPA (Tabela II e Figura 3). Este fato também é verificado através da observação dos pacientes que foram encaminhados para a UTI após a saída da SRPA. Uma porcentagem elevada dessas crianças não recebeu analgésicos (Tabela II).

O mito de que complicações, principalmente a depressão respiratória, associadas ao uso de opióides pioram o prognóstico de pacientes pediátricos pode contribuir para o menor uso desses fármacos para tratamento da dor pós-opera- tória. Estudo comparando a farmacocinética da morfina em adultos, crianças e recém-nascidos, a termo e prematuros, mostrou que após administração por via venosa, 30\% da morfina ligam-se a proteínas plasmáticas nos adultos e que somente $20 \%$ ligam-se a essas proteínas no recém-nascido. Esse aumento da porcentagem da droga livre permite que maior proporção da droga ativa atue no sistema nervoso central, justificando, em parte, os efeitos maiores da depressão respiratória. A meia vida de eliminação da morfina em adultos e crianças é de 3 a 4 horas, sendo consistente com o tempo de efeito analgésico. Em recém-nascidos menores que uma semana, esse tempo é mais que duplicado, e em crianças prematuras esse tempo é ainda maior. Desse modo, crianças menores que um mês podem apresentar níveis séricos mais altos de morfina, com declínio mais lento que os níveis séricos de adultos e das crianças maiores ${ }^{10-12}$.

Arealização de anestesia peridural sacral não é técnica muito praticada em nosso serviço nas crianças com menos de 1 ano de idade. No período de estudo, apenas 4 pacientes foram anestesiados com essa técnica. Desses 4 pacientes, apenas um recebeu analgésicos. Apesar de a anestesia peridural sacral mostrar-se técnica efetiva para promover analgesia pós-operatória ${ }^{13,14}$, nossa análise está prejudicada pelo pequeno número de casos em que tal técnica foi empregada nesse estudo.

Quanto ao uso de analgésicos pelos anestesiologista segundo a clínica cirúrgica, verificamos que as crianças das cirurgias cardíaca, torácica e otorrinolaringológicas foram as que menos analgésicos receberam (Tabela III). Não encontramos justificativa, considerando-se exclusivamente a clínica cirúrgica, para o comportamento observado.

Alistagem e a porcentagem dos fármacos empregados para alívio da dor pós-operatória é apresentada na tabela IV. A dipirona, isoladamente ou em associação com o tramadol ou a nalbufina, foi a droga mais freqüentemente utilizada.

$\mathrm{Na}$ literatura, observamos grande variedade de técnicas para promover analgesia pós-operatória. A cetamina, por via peridural sacral ou muscular, mostrou-se efetiva para analgesia pós-operatória após herniorrafia inguinal ${ }^{13}$. O cetorolaco, quando comparado com a analgesia através de anestesia peridural sacral, também foi satisfatório para promover analgesia após herniorrafia inguinal ${ }^{14}$. Também, para aliviar a dor após herniorrafia, o paracetamol tem sido bastante utilizado ${ }^{15}$. O bloqueio peridural sacral com anestésicos locais, associados ou não a outras drogas, principalmente opióides, é bastante utilizado com resultados bastante satisfatórios ${ }^{16,17}$.

Nesta análise retrospectiva, avaliando o uso de analgésicos para tratamento da dor pós-operatória em crianças menores que um ano, pudemos observar que utilizar analgésicos em crianças no período pós-operatório imediato não foi habitual, principalmente nas crianças menores e mais graves e em procedimentos cirúrgicos mais rápidos. O uso de dipirona, isoladamente, ou a associação dipirona/tramadol, foram as drogas analgésicas mais freqüentemente empregadas. 
É necessário a conscientização de todos os profissionais envolvidos com os cuidados dos pacientes pediátricos, sendo que a prevenção da dor pós-operatória é fundamental para um melhor resultado anestésico-cirúrgico, bem como para diminuir o sofrimento e o trauma associados ao período de permanência hospitalar.

\section{Postoperative Analgesia in Children Less than 1 Year of Age. A Retrospective Analy- sis}

Paulo do Nascimento Junior, TSA, M.D., Norma Sueli Pinheiro Módolo, TSA, M.D., Geraldo Rolim Rodrigues Junior, TSA, M.D.

\section{INTRODUCTION}

Pain is probably the most fearful symptom for human beings, but until recently, it had not been adequately addressed. It is one of the most common problems faced by individuals of all ages ${ }^{1}$.

Analgesics for postoperative pain in children, especially those less than 1 month of age, are still a very controversial subject. The resistance in prescribing adequate postoperative analgesia to pediatric patients and the reasons justifying such decision are discussed in the literature ${ }^{2,3}$. Some physicians still believe that neonates do not feel pain or have a different perception of pain. Others accept that very small children perceive and feel pain, but are more concerned with the risks of prescribing potent analgesics ${ }^{4}$.

A difficulty in adequately managing pediatric pain is the difficulty to obtain an accurate, objective and quantitative measure of pain, especially in neonates, infants and those below school age, because most available methods are not applied to all pediatric age brackets ${ }^{5}$.

Knowing that postoperative pain is still a major complication and reason for discomfort, especially in children, and aiming at knowing the profile of children not receiving immediate postoperative analgesics, both as therapeutic and prophylactic measures, this study has evaluated the use of analgesics from surgery completion to postanesthetic recovery unit discharge in children less than 1 year of age in the Hospital das Clínicas, Faculdade de Medicina de Bauru - UNESP.

\section{METHODS}

Based on the Anesthesiology Department database (Microsoft Access), a retrospective analysis was performed involving the period January/2000 to April/2001, of all anesthesias induced in children less than 1 year of age, submitted to different surgical procedures and referred to the PACU. Postoperative analgesia was evaluated considering the percentage of patients receiving analgesics from surgery completion to PACU discharge, the prevalence of analgesia according to age, gender, weight, anesthetic technique (general anesthesia or caudal anesthesia) and children's clinical status (ASA physical status), and patients' destination after PACU discharge, that is, ICU or ward.

To analyze drug prescription according to age, patients were divided in three age groups: children less than 1 month of age, between 1 and 6 months and from 6 months to 1 year. Medical specialties referring patients were also evaluated. Statistical analysis was performed using Chi-square and Fisher's Exact tests for frequency evaluation and Sudent's $t$ test for parametric variables comparison.

\section{RESULTS}

During the study period, 402 children less than 1 year of age were anesthetized. From these, $194(48.2 \%)$ were not medicated with analgesics and $208(51.8 \%)$ were (Table I). Table II and figure 1 show the distribution of patients, according to age, who received or not analgesics. Patients' distribution, according to gender and physical status (ASA), who received or not analgesics is illustrated in figures 2 and 3 , respectively, and table II. The evaluation of patients receiving or not analgesics, according to weight, anesthetic technique (general or caudal anesthesia), anesthesia duration and referral or not to ICU is shown in table II.

Table I - Total Number and Percentage of Patients Receiving or Not Analgesics from Surgery Completion to PACU Discharge

\begin{tabular}{lcc}
\hline & Number & $\%$ \\
\hline Without analgesia & 194 & 48.2 \\
With analgesics & 208 & 51.8 \\
\hline
\end{tabular}

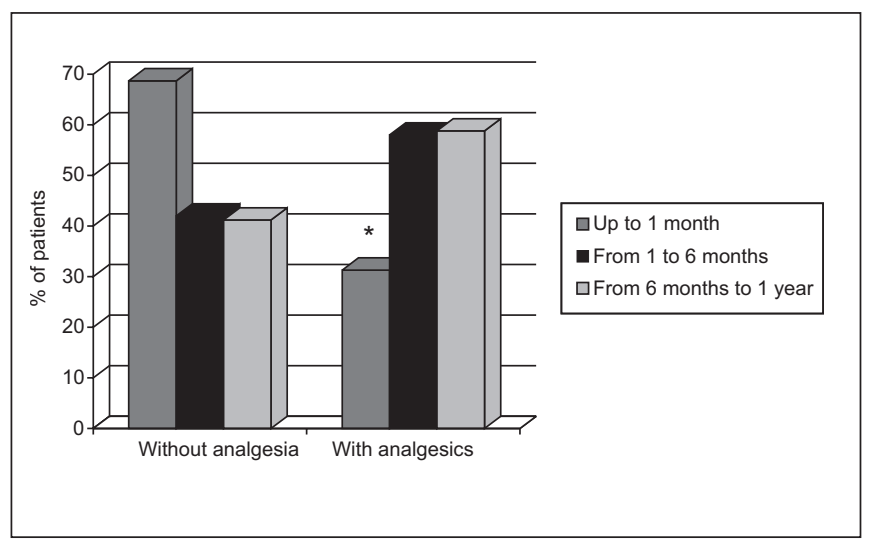

Figure 1 - Distribution of Patients in Percentages, According to Age, who Received or Not Analgesics from Surgery Completion to PACU Discharge

${ }^{*} p<0.01$ 
Table II - Evaluation of Patients Receiving or Not Analgesics from Surgery Completion to PACU Discharge, according to Age, Gender, Physical Status (ASA), Weight, Caudal Anesthesia Induction, Anesthesia Duration and Referral to ICU

\begin{tabular}{|c|c|c|c|}
\hline & W/o analgesia & With analgesics & Statistics \\
\hline Age & & & $p<0.01$ \\
\hline Up to 1 month $(n=99)$ & $68 / 99(68.7 \%)$ & $31 / 99(31.3 \%)$ & \\
\hline From 1 to 6 months $(n=126)$ & $53 / 126(42 \%)$ & $73 / 126(58 \%)$ & \\
\hline From 6 moths to 1 year $(n=177)$ & $73 / 177(41.2 \%)$ & $104 / 177(58.8 \%)$ & \\
\hline Gender & & & $p>0.05$ \\
\hline Male $(n=240)$ & $106 / 240(44.2 \%)$ & $134 / 240(55.8 \%)$ & \\
\hline Female $(n=162)$ & $88 / 162(54.3 \%)$ & $74 / 162(45.7 \%)$ & \\
\hline Physical status & & & $p<0.01$ \\
\hline ASA I $(n=187)$ & $69 / 187(36.9 \%)$ & $118 / 187(63.1 \%)$ & \\
\hline ASA $\|(n=113)$ & $56 / 113(49.5 \%)$ & $57 / 113(50.5 \%)$ & \\
\hline ASA III $(n=79)$ & $46 / 79(58.2 \%)$ & $33 / 79(41.8 \%)$ & \\
\hline ASA IV $(n=23)$ & $23 / 23(100 \%)$ & $0 / 23(0 \%)$ & \\
\hline Weight $(\mathrm{kg})^{*}$ & $6.7 \pm 3.1$ & $9.0 \pm 2.3$ & $p>0.05$ \\
\hline Anesthetic techniques & & & Não aplicada \\
\hline Caudal epidural $(n=4)$ & $3 / 4(75 \%)$ & $1 / 4(25 \%)$ & \\
\hline General $(n=398)$ & $191 / 398(48 \%)$ & $207 / 398(52 \%)$ & \\
\hline Anesthesia duration $(\mathrm{min})^{*}$ & $106 \pm 21$ & $130 \pm 38$ & $p<0.05$ \\
\hline Referral to ICU $(\mathrm{n}=119)$ & $93 / 119(78.1 \%)$ & $26 / 119(21.9 \%)$ & $p<0.01$ \\
\hline
\end{tabular}

* Values expressed in Mean \pm SD

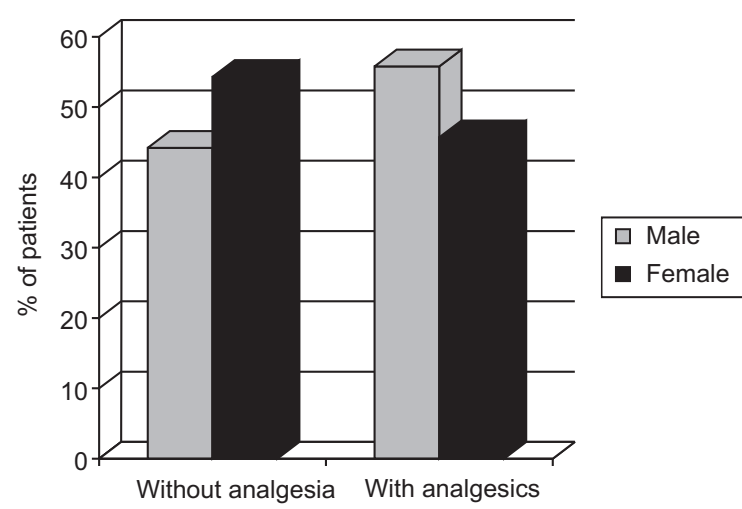

Figure 2 - Distribution of Patients in Percentages, According to Gender, who Received or Not Analgesics from Surgery Completion to PACU Discharge

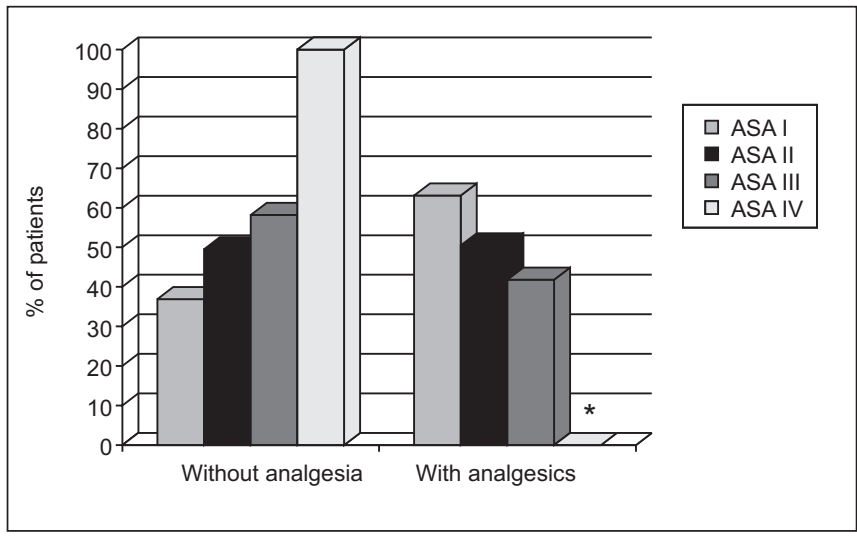

Figura 3 - Distribution of Patients in Percentages, According to Physical Status (ASA), who Received or Not Analgesics from Surgery Completion to PACU Discharge ${ }_{*} p<0.01$
Table III shows, according to the medical specialty referring the patients, the percentage of those receiving or not analgesics until PACU discharge.

Drugs prescribed for the 208 patients receiving analgesics are listed in table IV.

Table III - Patients Receiving or Not Analgesics from Surgery Completion to PACU Discharge, according to their Original Clinical Specialty

\begin{tabular}{lcc}
\hline & Without analgesia & With analgesics \\
\hline Pediatric surgery $(n=167)$ & $76(45 ., 5 \%)$ & $91(54.5 \%)$ \\
Neurosurgery $(n=66)$ & $27(41 \%)$ & $39(59 \%)$ \\
Ophthalmology $(n=44)$ & $21(47.7 \%)$ & $23(52.3 \%)$ \\
Urology $(n=27)$ & $7(26 \%)$ & $20(74 \%)$ \\
ENT $(n=27)$ & $19(70.3 \%)$ & $8(29.7 \%)$ \\
Orthopedics $(n=26)$ & $14(53.8 \%)$ & $12(46.2 \%)$ \\
Cardiac surgery $(n=21)$ & $16(76.1 \%)$ & $5(23.9 \%)$ \\
Plastic surgery $(n=17)$ & $8(47 \%)$ & $9(53 \%)$ \\
Chest surgery $(n=7)$ & $6(85.7 \%)$ & $1(14.3 \%)$ \\
\hline
\end{tabular}

Table IV - Drugs for Postoperative Analgesia from Surgery Completion to PACU Discharge, in Number of Patients and Percentages

\begin{tabular}{lcc}
\hline & Number & $\%$ \\
\hline Dipirone & 126 & 60.6 \\
Association dipirone/tramadol & 53 & 25.5 \\
Association dipirone/nalbuphine & 11 & 5.3 \\
Tramadol & 8 & 3.8 \\
Nalbuphine & 8 & 3.8 \\
Meperidine & 1 & 0.5 \\
Fentanyl & 1 & 0.5 \\
\hline
\end{tabular}

Revista Brasileira de Anestesiologia Vol. 52, № 6, Novembro - Dezembro, 2002 


\section{DISCUSSION}

Pain relief is the major goal of anesthesia. The anesthetic procedure should contemplate not only the perioperative period, but also the postoperative period. The evaluation of postoperative pain control in children has brought to light the current status of pain control in pediatrics ${ }^{6}$. The belief that children, especially neonates, are unable to feel pain has motivated surgeons and anesthesiologists not to prescribe postoperative drugs, especially opioids ${ }^{4}$.

The use of analgesics to treat already established postoperative pain or as a prophylactic measure to prevent any pain, is very important to determine well-being and comfort in the hospital.

Our study has shown that from 402 children less than 1 year of age, $194(48.2 \%)$ have not received analgesics from surgery completion to PACU discharge. Other authors have also reported a low percentage of postoperative analgesics in neonates, especially opioids ${ }^{7}$. When children are compared to adults, there are also many differences favoring adults with regard to the amount of postoperative analgesics prescription ${ }^{6}$.

Purcell-Jones et al., in 1988, have observed that in major surgical procedures performed in neonates, $48 \%$ of anesthesiologists would not prescribe opioids for postoperative pain control. For children up to 1 year of age, $37 \%$ of anesthesiologists would also not prescribe such drugs for postoperative pain relief. However, for minor surgical procedures, these percentages were $81 \%$ and $70 \%$, respectively, for the same age groups ${ }^{4}$. In similar analyses, other authors have observed a change in postoperative analgesia standards for pediatric patients favoring younger children ${ }^{8,9}$.

In our study with children receiving drugs for postoperative pain control, there has been a lower percentage of analgesics prescribed to children less than 1 month of age (Table II and Figure 1). Although without statistically significant differences, mean weight of patients not receiving analgesics was lower than of those receiving the drug (Table II). So, we observed the disfavoring of younger patients with regard to drugs prescription in the immediate postoperative period. As to patients' clinical conditions evaluated by physical status (ASA), there has been a lower use of analgesics in more severely ill children (ASA III and IV). No ASA IV patient received analgesics at surgery completion or in the PACU (Table II and Figure 3). This was also observed in patients referred to ICU after PACU discharge. A high percentage of those children have not received analgesics (Table II).

The myth that complications, especially respiratory depression, associated to the use of opioids worsen pediatric patients prognosis may contribute for the lower use of these drugs for postoperative pain control. Astudy comparing morphine pharmacokinetics in adults, children and term and premature neonates has shown that after intravenous administration, $30 \%$ of morphine is bound to plasma proteins in adults and only $20 \%$ are bound to these proteins in neonates. This increased percentage of free drug allows for a higher amount of active drug in the central nervous system, partly justifying major respiratory depression effects. Morphine elimination half-life in adults and children is 3 to 4 hours, being consistent with the analgesic effect duration. In neonates less than 1 week of age this time is more than doubled and in premature children it is even longer. So, children less than 1 week of age may present higher morphine plasma levels with a slower decline as compared to plasma levels of adults and older children ${ }^{10-12}$

Caudal anesthesia is not very widely used in our hospital in children less than 1 year of age. During the study period, only 4 patients were induced with this technique. From these 4 patients, only 1 received analgesics. Although caudal anesthesia being effective to promote postoperative analgesia ${ }^{13,14}$, our analysis was impaired by the small number of cases in which the technique was used.

As to the use of analgesics by anesthesiologists according to medical specialties, we have observed that cardiac, chest and ENT surgery children were those receiving less analgesics (Table III). No justification was found for this behavior, considering exclusively the surgical specialty.

A list with percentages of postoperative pain control drugs is shown in table IV. Dipirone alone or associated to tramadol was the most frequent drug.

There are several techniques in the literature to promote postoperative analgesia. Caudal or muscular ketamine has shown to be effective for post inguinal hernioplasty analgesia ${ }^{13}$. Ketorolac, as compared to caudal analgesia, was also effective in promoting post inguinal hernioplasty analgesia ${ }^{14}$. Paracetamol has also been widely used for post hernioplasty pain relief ${ }^{15}$. Caudal epidural block with local anesthetics associated or not to other drugs, especially opioids, is widely used with very satisfactory results ${ }^{16,17}$.

In this retrospective evaluation of analgesics for postoperative pain control in children less than 1 year of age, it was observed that analgesics for children in the immediate postoperative period is not a routine, especially in younger and more severely ill children and in shorter surgical procedures. Dipirone, or the association dipirone/tramadol, where the most widely used drugs.

All professionals involved in pediatric care must be aware that postoperative pain control is paramount for a better surgical-anesthetic result, as well as for decreasing suffering and trauma associated to hospital stay.

\section{REFERÊNCIAS - REFERENCES}

01. Barbosa SMM, Dias MHP, Santos E et al - A criança e a dor: considerações sobre o tratamento da dor em pediatria. Rev Dor, 2000;2:13-18.

02. Bray RJ - Post-operative analgesia provided by morphine infusion in children. Anaesthesia, 1983;38:1075-1078.

03. Mather L, Mackie J - The incidence of post-operative pain in children. Pain, 1983;15:271-282.

04. Purcell-Jones G, Dormon F, Sumner E - Paediatric anaesthetists' perceptions of neonatal and infant pain. Pain, 1988;33: 181-187.

05. Lloyd-Thomas AR - Pain management in paediatric patients. $\mathrm{Br}$ J Anaesth, 1990;64:85-104. 
06. Beyer JE, DeGood DE, Ashley LC et al - Patterns of postoperative analgesic use with adults and children following cardiac surgery. Pain, 1983;17:71-81

07. Purcell-Jones G, Dormon F, Sumner E - The use of opioids in neonates. A retrospective study of 933 cases. Anaesthesia, 1987; 42:1316-1320.

08. Lima J, Lloyd-Thomas AR, Howard RF et al - Infants and neonatal pain: anaesthetists' perceptions and prescribing patterns. $\mathrm{Br}$ Med J, 1996;313:787.

09. McLaughlin CR, Hull JG, Edwards WH et al - Neonatal pain: a comprehensive survey of attitudes and practices. J Pain Symptom Manage, 1993;8:7-16.

10. Lloyd-Thomas AR - Pain management in paediatric patients. $\mathrm{Br}$ J Anaesth, 1990;64:85-104.

11. Weisman SJ, Schechter N - The management of pain in children. Pediat Review, 1991;12:237-243.

12. Swafford L, Allen D - Pain relief in pediatric patient. Med Clin North Am, 1968;52:131-136.

13. Koinig $H$, Marhofer $P$, Krenn $C G$ et al - Analgesic effects of caudal and intramuscular $\mathrm{S}(+)$-ketamine in children. Anesthesiology, 2000;93:976-980.

14. Splinter WM, Reid CW, Roberts DJ et al - Reducing pain after inguinal hernia repair in children: caudal anesthesia versus ketorolac tromethamine. Anesthesiology, 1997;87:542-546

15. Wiener ES, Touloukian RJ, Rodgers BM et al - Hernia survey of the section on surgery of the American Academy of Pediatrics. J Pediatr Surg, 1996;31:1166-1169.

16. Khalil S, Campos C, Farag AM et al - Caudal block in children: ropivacaine compared with bupivacaine. Anesthesiology, 1999;91:1279-1284

17. Girotra S, Kumar S, Rajendran KM - Postoperative analgesia in children who have genito-urinary surgery. A comparison between caudal buprenorphine and bupivacaine. Anaesthesia, 1990;45:406-408.

\section{RESUMEN}

Nascimento Jr P, Módolo NSP, Rodrigues Jr GR - Analgesia Pós-Operatoria para Niños con Menos de 1 Año. Análisis Retrospectivo

Justificativa y Objetivos - El dolor pós-operatorio continua siendo una de las principales complicaciones pós-operatorias y motivo de incomodidad, principalmente en niños. El objetivo de este estudio fue evaluar el uso de analgésicos desde el término de la cirugía hasta el alta de la sala de recuperación pós-anestésica (SRPA), como medida terapéutica o profiláctica, para niños con menos de 1 año de edad.

Método - Utilizando el banco de datos del Departamento de Anestesiologia, fue realizada un análisis retrospectivo, envolviendo el período de enero/2000 a abril de 2001, de las anestesias de niños menores de 1 año de edad sometidos a procedimientos quirúrgicos diversos, evaluando aspectos relacionados a la analgesia pós-operatoria.

Resultados - En el período de estudio, fueron anestesiados 402 niños menores de un 1 año, siendo que 194 (48,2\%) no recibieron analgésicos y 208 (51,8\%) recibieron. Con relación al uso o no de analgésicos, lo que se sigue fue observado: Sin analgésicos: (1) Edad: hasta 1 mes, 68/99; entre 1 y 6 meses, 53/126; entre 6 meses y 1 año, 73/177. (2) Peso: 6,7 $\pm 3,1 \mathrm{~kg}$ (3). Sexo: masculino, 106/240; feminino, 88/162. (4) Estado físico ASA: ASA I, 69/187; ASA II, 56/113; ASA III, 46/79; ASA IV, 23/23. (5) Anestesia peridural sacral: 3/4. (6) Tiempo de anestesia: $106 \pm 32$ minutos. (7) Encaminamiento para unidad de terapia intensiva (UTI): 93/119. Uso de analgésicos: (1) Edad: hasta 1 mes, 31/99; entre 1 y 6 meses, 73/126; entre 6 meses y 1 año, 104/177. (2) Peso: $9 \pm 2,3 \mathrm{~kg}$. (3) Sexo: masculino, 134/240; femenino, 74/162. (4) Estado físico ASA: ASA I, 118/187; ASA II, 57/113; ASA III, 33/79; ASA IV, 0/23. (5) Anestesia peridural sacral: 1/4. (6) Tiempo de anestesia: $130 \pm$ 38 minutos. (7) Encaminamiento para UTI: 26/119. Los fármacos empleados para promover analgesia fueron: dipirona $(60,6 \%)$, dipirona + tramadol $(25,5 \%)$, dipirona + nalbufina $(5,3 \%)$, tramadol $(3,8 \%)$, nalbufina $(3,8 \%)$, meperidina $(0,5 \%)$ y fentanil $(0,5 \%)$.

Conclusiones - Utilizar analgésicos en niños desde el término de la cirugía hasta el alta de la SRPA no fue habitual, principalmente en niños menores y más graves y en procedimientos quirúrgicos más rápidos. El uso de dipirona, aisladamente, o la asociación dipirona/tramadol, fueron las drogas analgésicas más frecuentemente empleadas. 